\title{
Mitigation of the kink instability in the energy recovery linac based electron-ion collider
}

\author{
Yue Hao, Vladimir N. Litvinenko, and Vadim Ptitsyn \\ Brookhaven National Laboratory, Upton, New York 11973, USA, and Department of Physics and Astronomy, \\ Stony Brook University, Stony Brook, New York 11794, USA
}

(Received 26 April 2013; published 22 October 2013)

\begin{abstract}
Beam-beam effects in eRHIC, the proposed energy recovery linac (ERL)-based electron-ion collider (EIC) at BNL, have several unique features distinguishing them from those in hadron and lepton colliders. Taking the advantage of the fact that the electron beam is used only once, we expect the luminosity to be 10 times that of a ring-ring collision scheme with similar parameters. However, without instituting proper treatments, the quality of electron and hadron beams can undergo degradation or even beam loss, driven by the beam-beam interactions. Kink instability is one of the most important collective effects of the ion ring due to the special beam-beam interaction in an ERL-based EIC. In this article, we present novel treatments for kink instability in ERL-based EICs including a scheme dedicated to suppressing instability.

DOI: 10.1103/PhysRevSTAB.16.101001

PACS numbers: $29.20 .-\mathrm{c}$
\end{abstract}

\section{INTRODUCTION}

The main advantage of an energy recovery linac (ERL) based electron-ion collider (EIC) compared with a ringring collider is the higher achievable luminosity of the former [1]. In an ERL-based EIC, which we also call a linac-ring scheme, the electron bunch collides only once with the ion bunch and thereafter is recycled. Hence, the beam-beam parameter for the electrons in ERL scheme can exceed by a large margin (as in Table I) that permissible for electron circulating in a ring. While the beam-beam parameter for the ions remains the same in both schemes, the luminosity achieved in the linac-ring collision scheme exceeds that of the ring-ring collider scheme by 1 to 2 orders of magnitude [1]. Table I lists the range of parameters for eRHIC, the ERL-based EIC, proposed at Brookhaven National Laboratory. From the table, the range of beam-beam parameter for the electron beam is much larger than the achieved value in an lepton collider $(\sim 0.1)$, while the parameters for the ion/proton beam are limited to the proven value in RHIC operation. Hereafter, we use the term ion to describe both the proton and ion beams.

In the new luminosity regime of the ERL-based EIC, the electron beam is subjected to a very strong beam-beam effects that create a new set of beam dynamics effects. First, the electron beam experiences significant disruption and mismatch due to the beam-beam interaction [2]. In addition, the collective motion of the electron beam inside the ion beam during their collision can cause a new headtail-type of instability, as was observed in earlier simulations [3] and termed "kink instability."

Published by the American Physical Society under the terms of the Creative Commons Attribution 3.0 License. Further distribution of this work must maintain attribution to the author(s) and the published article's title, journal citation, and DOI.
In this paper, we review the mechanism of the kink instability and derivation of its threshold. We also discuss methods of mitigating it. Specifically, we detail a unique feedback scheme that acts only on the electron beam to suppress the emittance growth of the ion beam caused by this instability.

\section{REVIEW OF KINK INSTABILITY}

If the centroids of the ion or the electron beam are slightly offset in the interaction region (IR), the electron beam trajectory will undergo transverse oscillations inside the ion beam. In practice, the electron bunch is much shorter than the ion bunch. Since both colliding bunches are ultrarelativistic, we can describe the collision as that of a thin (infinite short) electron bunch colliding at longitudinal position $s$ with that portion of the ion bunch located $z=2 s$ with respect to its center. In the eRHIC, this assumption is a valid one since the electron bunch is at least 40 times shorter than the ion bunch, if an advanced cooling method is applied to the ion beam. The motion of an electron can be approximately described by a linear beam-beam force, provided that transverse offset is small compared with the beam size of both beams:

TABLE I. eRHIC parameter range of interest.

\begin{tabular}{lc}
\hline \hline Parameters & Range \\
\hline Electron beam energy $(\mathrm{GeV})$ & $5-30$ \\
Ion beam energy $(\mathrm{GeV})$ (proton) & $50-250$ \\
Electron beam disruption & $4-150 / 0.3-10$ \\
$\quad$ parameter/beam-beam parameter & \\
Ion beam-beam parameter & $<0.015$ \\
Ion bunch length $(\mathrm{cm})$ & $8.3-20$ \\
Electron bunch length $(\mathrm{cm})$ & $0.2-0.4$ \\
Electron and ion $\beta^{*}(\mathrm{~cm})$ & $5-20$ \\
Ion synchrotron tune & 0.004 \\
\hline \hline
\end{tabular}




$$
x_{e}^{\prime \prime}+k^{2}(s)\left[x_{e}-\bar{x}_{i}(s, z=2 s)\right]=0,
$$

where $\bar{x}_{i}(s, z)$ is the local transverse offset of the ion bunch centroid, which is a function of the longitudinal coordinate $s$. The beam-beam interaction strength $k(s)$ has the following form:

$$
k^{2}(s)=\frac{2 Z N_{i} r_{e}}{\sigma_{i x}^{2} \gamma_{e}} \lambda(z=2 s)=\frac{2 \lambda(z=2 s)}{f_{e}}=\frac{2 d_{e} \lambda(z=2 s)}{\sigma_{i z}},
$$

where $Z$ and $N_{i}$ are the ion charge and bunch intensity respectively, $\sigma_{i x / z}$ are the rms transverse and longitudinal ion beam size, $\lambda(z)$ is the ion longitudinal distribution, $r_{e}$ is the classical radius of the electron, $f_{e}=4 \pi \xi_{e} / \beta_{e}^{*}$ is the beam-beam focal length for the electron beam, and $d_{e}=$ $\sigma_{i z} / f_{e}$ is the disruption parameter [2]. Let us consider the ion bunch confined within $z= \pm L$. For a Gaussian ion bunch, it is sufficient to set $L=5 \sigma_{i z}$. Then without the limitation of generality, we can assume that the initial conditions for electron beam before the collision can be set at zero, i.e. $\bar{x}_{e}(L / 2)=0$ and $\bar{x}_{e}^{\prime}(L / 2)=0$. In this case, the electron bunch travels along the $-\hat{s}$ direction, so that the acquired offset of the electron beam at position $s$ depends on the ion beam displacement in the region $[s, L]$, which it samples.

The equation for the motion of the electron bunch centroid $\bar{x}_{e}(s)$ is obtained by averaging Eq. (1). Since an ion at the $z$ location within the ion bunch encounters a short electron bunch at $s=z / 2$, its motion can be described approximately by a linear kick, assuming a small offset:

$$
x_{i}^{\prime \prime}(s, z)+K^{2}(s) x_{i}=\delta\left(s-\frac{z}{2}\right) \frac{x_{i}-\bar{x}_{e}(s)}{f_{i}},
$$

where $K(s)$ denotes the ion ring's focusing strength, and $f_{i}$ is the beam-beam focal length for the ion bunch. We note that the longitudinal displacement $z$ of the ion undergoes synchrotron oscillation. The beam-beam focusing force on the right-hand side of the equation is proportional to the offset difference of the ion beam and electron beam's centroid. The latter, according to the Eq. (1), is a function of the ion bunch centroid offsets in front of the ion. Therefore, the electron beam introduces an effective wakefield that couples the kick of the ion with the offset of the ions ahead of it.

A simplified case was considered in Ref. [4] when both beams have rigid transverse beam size and the ion bunch has a uniform longitudinal distribution, i.e. $k^{2}(s)=$ $1 /\left(L f_{e}\right)$. In this case Eq. (1) has the simple solution:

$$
\bar{x}_{e}=k \int_{s}^{L / 2} \bar{x}_{i}\left(s^{\prime}, z=2 s^{\prime}\right) \sin k\left(s-s^{\prime}\right) d s^{\prime},
$$

and the wakefield is a sinusoidal function:

$$
W\left(s-s^{\prime}\right) \sim k \sin k\left(s-s^{\prime}\right) H\left(s^{\prime}-s\right) .
$$

Here, $H(x)$ is the Heaviside step function. The threshold for this sinusoidal wakefield [Eq. (5)] was evaluated in the same work [4]. However, the longitudinal distribution of the ion bunch usually is nonuniform, and, frequently is modeled by a Gaussian or an elliptical distribution. Therefore, in general, the wakefield cannot be obtained analytically, and requires computer simulations. In a simulation code, the long ion bunch is cut into a number of short longitudinal slices. We can calculate the transverse kick $\Delta x^{\prime}(s)$ at $s$ due to an offset set in a slice at $s^{\prime}$ of $\Delta x\left(s^{\prime}\right)$, and obtain the wakefield, which is defined as

$$
W\left(s, s^{\prime}\right)=\frac{\gamma_{i} \Delta x^{\prime}(s)}{Z^{2} N_{i b} r_{i} \Delta x\left(s^{\prime}\right)}
$$

where $N_{i b}$ is the number of ions in the slice and $r_{i}$ is the classical radius of the ion. With this definition, we may take into consideration several important effects, such as the hourglass effect, the arbitrary ion beam longitudinal distribution, and the variations in the size of electron beam during the interaction, usually referred as "pinch effect." Figure 1 illustrates two examples of the wakefield that we
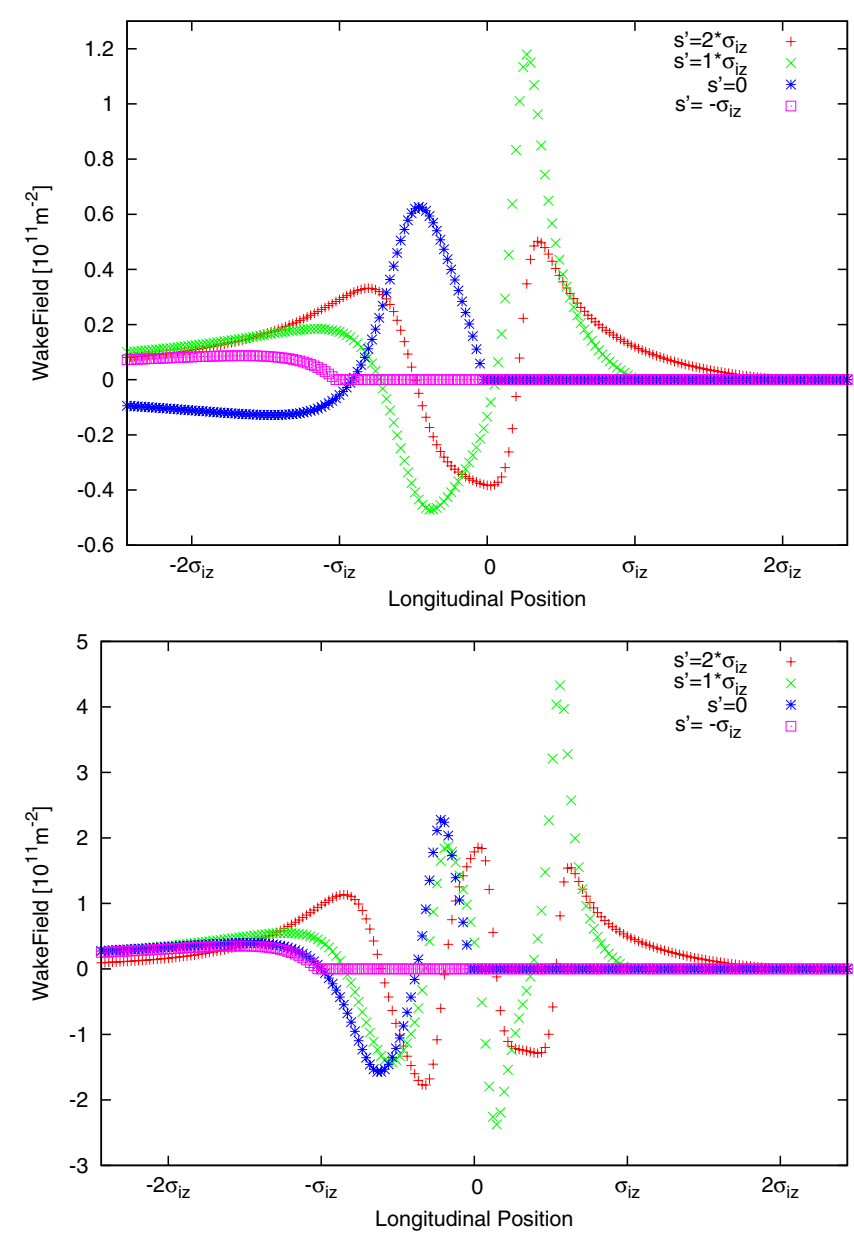

FIG. 1. Two examples of the kink wakefield with the beambeam parameter of the ion beam $\xi_{p}=0.015$. Top: The electron beam disruption parameter $d_{e}=27$; bottom: $d_{e}=108$. In both figures, the electron bunch travels from the positive $s$ to negative. $s^{\prime}$ denotes the location of the introduced offset. 
calculated from the beam-beam code EPIC [5]. For this calculation, we assumed a 6D Gaussian distribution for the ion bunch.

Before introducing the results of the simulating code, we employed alternative matrix methods than Ref. [4] to explore the instability threshold, especially for the case of large disruption parameter, i.e., when the electron bunch executes one or more betatron oscillations during its collision with the ion bunch. Starting from a simple twoparticle model [6,7], we can assess the threshold of strong head-tail instability based on a constant wakefield. In this model two macroparticles represent the ion beam, both carrying $N_{i} / 2$ charges and residing at $\pm_{0}$ respectively; the one at the negative longitudinal position is the trailing particle and experiences the wakefield due to the beambeam interaction and the orbit offset of the heading counterpart. After half synchrotron period, the two macroparticles exchange their positions. Assuming particle 2 is the trailing one at the beginning, the kick strength of the wakefield is

$$
x_{2}^{\prime}=\frac{z_{0}}{2 f_{e} f_{i}} x_{1} \equiv \kappa x_{1} .
$$

Therefore the transverse matrix for two particles is

$$
M_{N s}=(M \bar{K})^{N_{s} / 2}(M K)^{N_{s} / 2},
$$

where $M$ is the $4 \times 4$ transverse transformation one-turn matrix for two particles, $N_{s}$ is the reciprocal of the synchrotron tune and the matrices $K$ and $\bar{K}$ represent the wakefield kick:

$$
K=\left(\begin{array}{cccc}
1 & 0 & 0 & 0 \\
0 & 1 & 0 & 0 \\
0 & 0 & 1 & 0 \\
\kappa & 0 & 0 & 1
\end{array}\right) ; \quad \bar{K}=\left(\begin{array}{cccc}
1 & 0 & 0 & 0 \\
0 & 1 & \kappa & 0 \\
0 & 0 & 1 & 0 \\
0 & 0 & 0 & 1
\end{array}\right) \text {. }
$$

We rewrite the matrix $M K$ and $M \bar{K}$ in its Jordan decomposition form $M K=P D P^{-1}$ and $M \bar{K}=\bar{P} \bar{D} \bar{P}^{-1}$, so that the power of the matrix can be written as

$$
M_{N s}=\bar{P} \bar{D}^{N_{s} / 2} \bar{P}^{-1} P D^{N_{s} / 2} P^{-1} .
$$

Reasonably assuming that $N_{s} \gg 1$ and $N_{s} \gg \cot (2 \pi \phi)$; $\phi=2 \pi \nu_{x}$, where $\nu_{x}$ is the transverse tune of the ion ring, the $M_{N s}$ can be simplified as

$$
M_{N s}=\left(\begin{array}{cc}
1-\left(\frac{\kappa N_{s} \beta}{4}\right)^{2} Y\left(N_{s} \phi\right) & \frac{\kappa N_{s} \beta}{4} Y\left(N_{s} \phi-\frac{\pi}{2}\right) \\
\frac{\kappa N_{s} \beta}{4} Y\left(N_{s} \phi-\frac{\pi}{2}\right) & Y\left(N_{s} \phi\right)
\end{array}\right)
$$

and

$$
Y=\left(\begin{array}{cc}
\cos \phi+\alpha \sin \phi & \beta \sin \phi \\
-\gamma \sin \phi & \cos \phi-\alpha \sin \phi
\end{array}\right)
$$

The threshold can be derived from eigenvalues of the matrix $M_{N s}$, which has a simple form:

$$
\xi_{i} d_{e}<\frac{4 z_{0} \nu_{s}}{\pi \sigma_{i z}} .
$$

Here, $\xi_{i}$ is the beam-beam parameter for the ion beam, and $d_{e}$ is the disruption parameter of the electron beam. For ions with $z_{0}=\sigma_{i z}$ the threshold simply reads $\xi_{i} d_{e}<$ $4 \nu_{s} / \pi$.

In this simple model we ignore details of the oscillating wakefield, illustrated in Fig. 1 or the sinusoidal form [Eq. (5)]. Therefore, it is an approximation suitable only for a small disruption parameter when the electrons execute much less than a full oscillation inside the ion bunch. An $N$-particle model using the circulant matrix method [8] allows us to the next level.

According to this method, the total matrix, representing the synchrotron-betatron motion, can be constructed as

$$
M_{\mathrm{sb}}=M_{c} \otimes M_{b},
$$

where $M_{b}$ is the $2 \times 2$ betatron one-turn map, $\otimes$ denotes outer product of the matrices and $M_{c}$ is a $N \times N$ circulant matrix with its elements defined as

$$
M_{c}(i, j)=\frac{\sin \left(\psi_{i, j} N\right)}{N \sin \left(\psi_{i, j}\right)},
$$

where

$$
\psi_{i, j}=\pi\left[\nu_{s}-(N-i+j) / N\right]
$$

and $\nu_{s}$ represents the synchrotron tune.

In this model, the $N$ macroparticles display same amplitude of synchrotron oscillation and are separated by equal longitudinal phase spacing. Each macroparticle is characterized by its own transverse offset, while the circulant matrix $M_{c}$ modifies the macroparticle offsets (and their slopes) caused by the synchrotron motion. Thus, the model represents a hollow bunch in the longitudinal phase space, and realizes the transverse offsets among the adjacent macroparticles. When $N=2 m+1$ is an odd number, the eigenvalues of the synchrotronbetatron matrix $M_{\mathrm{sb}}$ give the transverse tune $\nu_{\beta}$, and its $2 m$ sidebands: $\nu_{\beta}, \nu_{\beta} \pm \nu_{s}, \ldots, \nu_{\beta} \pm m \nu_{s}$. To include the effect of ion-electron beam-beam interaction, we extend the $2 N \times 2 N$ matrix $M_{\mathrm{sb}}$ to the $(2 N+2) \times(2 N+2)$ matrix $M_{t}$,

$$
M_{t}=\left(\begin{array}{cc} 
& 0 \\
M_{\mathrm{sb}} & \vdots \\
& 0 \\
0 \cdots 0 & \varnothing
\end{array}\right) \cdot M_{k},
$$

where $M_{k}$ denotes the beam-beam interaction between the electron and ion beams. $M_{k}$ is found by multiplying the matrices of the linear beam-beam interaction, describing the interactions of the electron bunch with each of the ion 

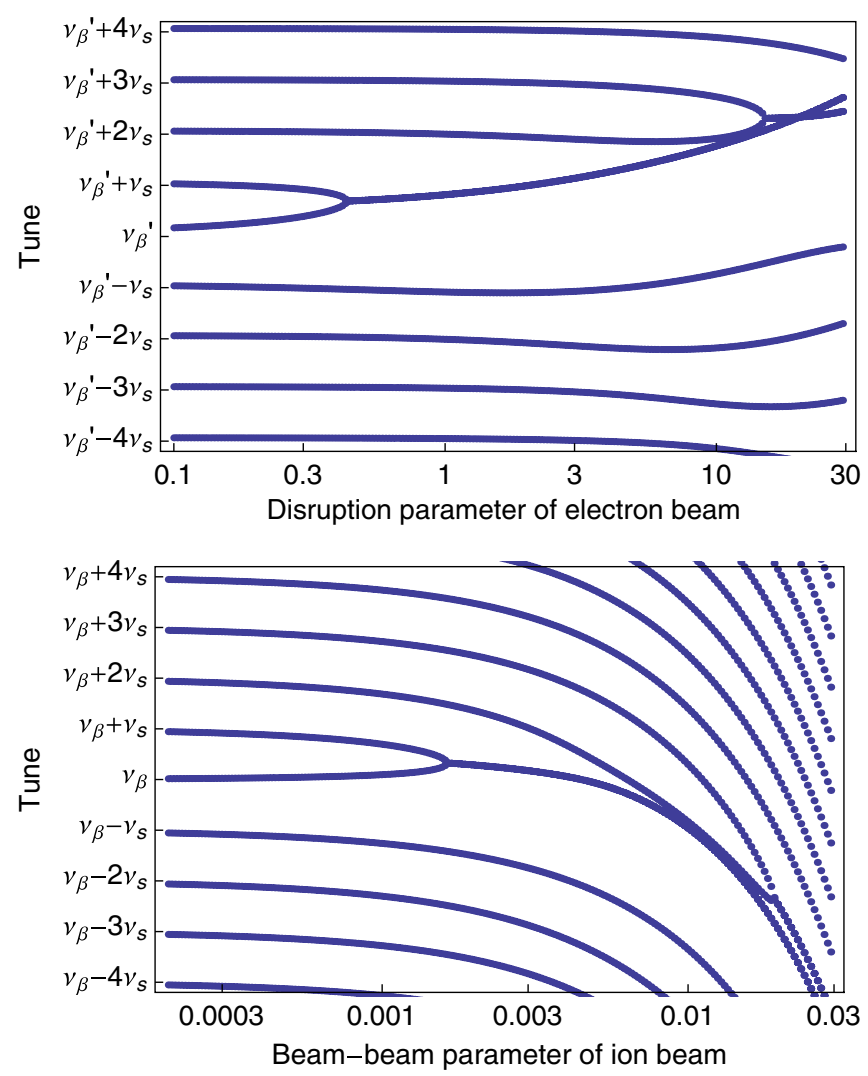

FIG. 2. Frequency contents of the matrix $M_{t}$ as a function of the disruption parameter of the electron bunch with $\xi_{i}=0.015$ (top) and the beam-beam parameter of the ion bunch with $d_{e}=$ 10 (bottom). In the vertical axis, $\nu_{\beta}$ and $\nu_{s}$ denote the betatron and synchrotron tunes respectively, $\nu_{\beta}^{\prime}=\nu_{\beta}-\xi_{i}$ is the betatron tune including the beam-beam tune shift.

macroparticles, and the drift matrices between adjacent macroparticles. $\varnothing$ denotes a two-by-two transverse matrix for the electron beam. Since on every turn the electron bunch is fresh and is discarded after collision, all elements of $\varnothing$ are zero.

The instability threshold can be derived, as is common, by the appearance of merging of the betatron frequency and its sidebands, derived from the eigenvalues of matrix $M_{t}$. Figure 2 illustrates the frequency contents extracted from its eigenvalues. The first mode merging corresponds to the threshold of the strong head-tail instability. Figure 3 compares the threshold dependence on the ion beam-beam parameter and on the electron disruption parameter for different models. The synchrotron tune is fixed at 0.004 . In a dual logarithmic scale, the two-particle model predicts that the threshold relationship [Eq. (12)] is a straight line. It agrees with the multiparticle model only when the electron disruption parameter $d_{e}$ is small $(\leq 1)$. The relationship largely deviates from the simple form when $d_{e}$ approaches 10. The maximum allowed ion beam-beam parameter $\xi_{i}$, at higher values of the disruption parameter decreases much more slowly, as $d_{e}$ increases, and forms several local peaks and valleys. The oscillating pattern comes from the fact

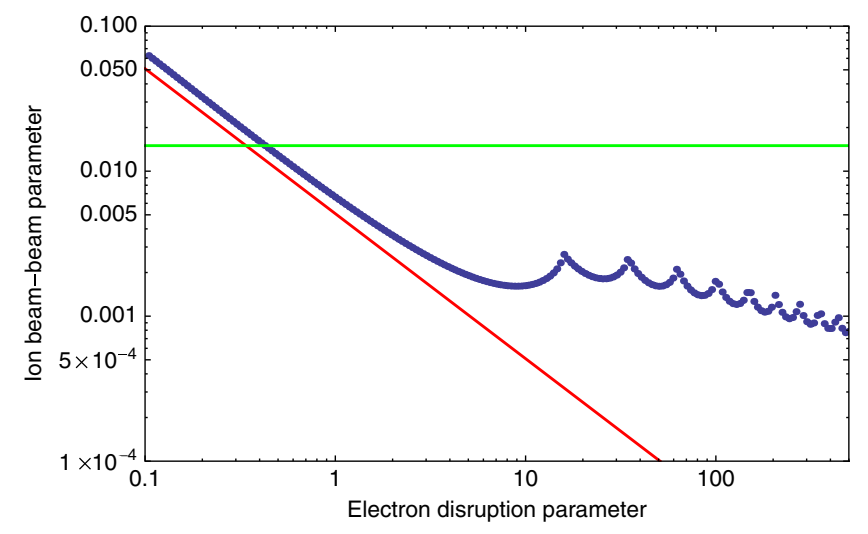

FIG. 3. The threshold of kink instability, with choice of the synchrotron tune 0.004 . The blue dots denote the threshold calculated from 51 macroparticles circulant matrix method. The red line represents the simple threshold form from Eq. (12) with $z_{0}=\sigma_{i z}$. The green line corresponds to $\xi_{i}=$ 0.015 , which is the design beam-beam parameter of the ion bunch in eRHIC.

that the electron bunch finishes about $\sqrt{d_{e}} / 4$ times full oscillation in the opposing ion beam [2]. It is worthwhile to note that he number of the macroparticle $N_{p}$ must be large enough to reflect the number of oscillation of the electron beam. For $d_{e}=150$, the largest disruption parameter considered in this paper, we found the $N_{p}>21$ give the converged results. All results shown in this section are calculated with $N_{p}=51$.

From Table I and Fig. 3, we anticipate that the eRHIC parameters will be beyond the kink instability threshold and that countermeasures must be considered. However, both matrix models we employed are based on a linearized beam-beam interaction, rigid electron beam sizes, the zero betatron tune spread of the ion beam, a special ion bunch longitudinal distribution, and a simplified longitudinal motion. Therefore, simulation codes are necessary to include these essential missing effects. The code, named EPIC [5,9], is a dedicated code for various beam-beam effects in linacring based colliders. We will also use it to demonstrate different methods of mitigating the kink instability.

We note that the phase advance of an individual ion between IP $(s=0)$ and the actual collision point $(s=z / 2)$ is responsible for head-tail instability without threshold, since the betatron motion and synchrotron motion are coupled by the phase advance with $z$ dependence, which is explored in Ref. [10]. Provided that this effect is included, the matrix $M_{t}$ always has growing mode whose eigenvalue's amplitudes are greater than 1 . However, if we employ a nonlinear beam-beam field in simulation, we do not observe the emittance growth of the ion beam due to instability below the strong head-tail threshold of $d_{e}$ or $\xi_{i}$. Because of the tune spread introduced by the nonlinear beam-beam field, instead, the threshold of instability is always larger than the predicted value using linear the beam-beam model. Therefore, we still consider kink 
instability in linac-ring colliders is dominated by the transverse mode coupling instability (strong head-tail instability).

\section{SIMULATION RESULTS OF THE INSTABILITY AND SUPPRESSION WITH LANDAU DAMPING}

We used the simulation code EPIC to explore the beambeam collisions of the electron and ion beams including various effects beyond the linear model. Those effects include the betatron amplitude dependent tune spread due to the beam-beam interaction, and the variation of the transverse beam sizes of electrons and ions throughout the collision region due to the beta-function profile and the electron pinch effect. The beam parameters shown in Table I have been used for the simulations. The beam-beam parameter of the ion beam is kept at 0.015 and the disruption parameter of the electron beam is adjusted by varying its energy. The effects of chromaticity and the synchrotron motion due to the rf system also are included. In a nominal RHIC operation, the chromaticity of both transverse planes is set to about +2 units at the beam store to suppress the coherent effect due to the various wakefields in the RHIC rings. In the presence of this chromaticity, we simulated the kink instability induced by the electron-ion collision near the current working point of RHIC $(0.675,0.67)$. Certainly the chromaticity must be positive since the nominal store energy of RHIC is above the transition energy.

In our simulation, we track the ion beam emittance growth due to the kink instability. Figure 4 shows the emittance growth associated with the kink instability at different disruption parameters of the electron beam. Even with the lowest one, $d_{e}=5$, the system is unstable at +2 chromaticity, although then the emittance growth is much

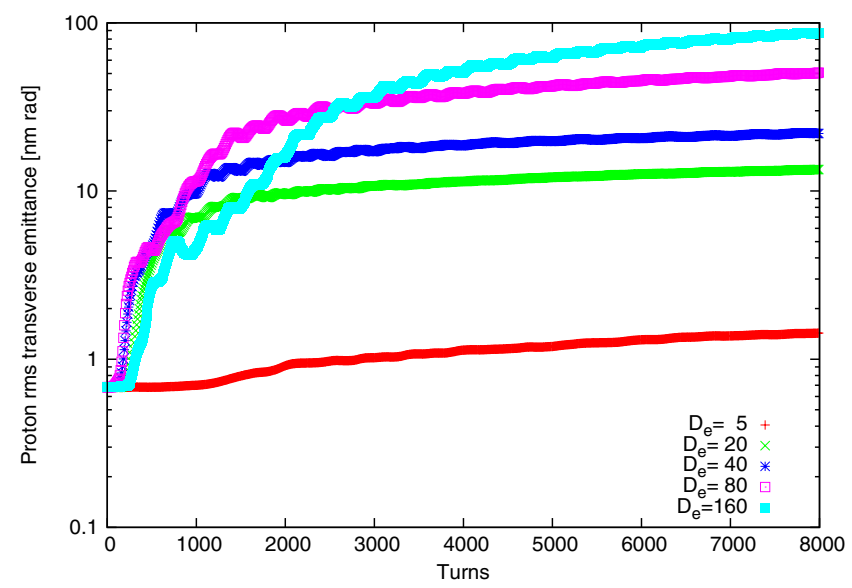

FIG. 4. The proton beam emittance growth due to the kink instability at different disruption parameters with the chromaticity of both transverse directions set at +2 units, and the beambeam parameter of the proton beam at 0.015 . The scale of the $y$ axis is a logarithm to make the emittance growth of the $d_{e}=5$ case visible. less that those with higher $d_{e}$. The emittance growth of the kink instability rises from the initial numerical noise of the macroparticle. To get converged results, we use 100 and $10 \mathrm{~K}$ macroparticles to represent the ion and the electron beam, respectively. The ion beam has 50 longitudinal slices. We observed that at least 30 longitudinal slices are required to yield converged results.

A common approach to suppressing the kink instability is via Landau damping using the proper transverse tune spread. The nonlinear force of the beam-beam interaction inevitably introduces a transverse tune spread, however, it is insufficient to suppress the instability according to the results shown in Fig. 4. We have investigated the possibility of suppressing the kink instability by increasing transverse tune spread via adjusting the chromaticity. The results are presented in Fig. 5. The top left figure (red, green, and blue dots) plots the suppression of the emittance growth at $d_{e}=5$. A large chromaticity, 12 units, is required to fully suppress the emittance growth caused by kink instability. For a higher disruption parameter, $d_{e}=10$, the required chromaticity for suppressing instability is 16 , as shown in the top right figure. In the case of a higher disruption parameter, it requires even unreasonable chromaticity.

Adding a bunch-by-bunch transverse feedback system (transverse damper) into RHIC is a possible way to lower the required chromaticity for suppressing the emittance growth. Here we will explore the required chromaticity of the ion ring in the presence of an ideal transverse bunchby-bunch feedback system. For such a damper in RHIC, we assume its damping time of such a system is 50 to 100 turns. For hadron machines, this assumption is reasonable since a shorter damping time may introduce unacceptable noise level to the ion beam.

Our finding from the simulation shows that, at disruption parameter $d_{e}=5$, the feedback system can suppress the kink instability with only slightly increased chromaticity ( $<4$ units). In the case of $d_{e}=10$, the required chromaticity is also reduced due to the presence of the transverse damper. The simulation results reveals that the minimum chromaticity to damp the instability is 12 when the damping time of the transverse damper is 100 turns, compared with +16 units without it (bottom left of Fig. 5). If the gain of the damper increases and the damping time can reach 50 turns, then, the minimum chromaticity required is eight units. With the same damper gain, when $d_{e}=20,+12$ units of chromaticity are needed (bottom right of Fig. 5).

We learned that, even with the presence of the transverse damper, the minimum chromaticity for suppressing the kink instability is much larger than the nominal chromaticity ( 2 units) in the current RHIC operation, except for the case of lowest disruption parameter $\left(d_{e} \sim 5\right)$. The large tune spread associated with those chromaticities overlaps nonlinear resonances which can lead to the beam lifetime problems. Therefore, a dedicated feedback system is desired as a countermeasure. 

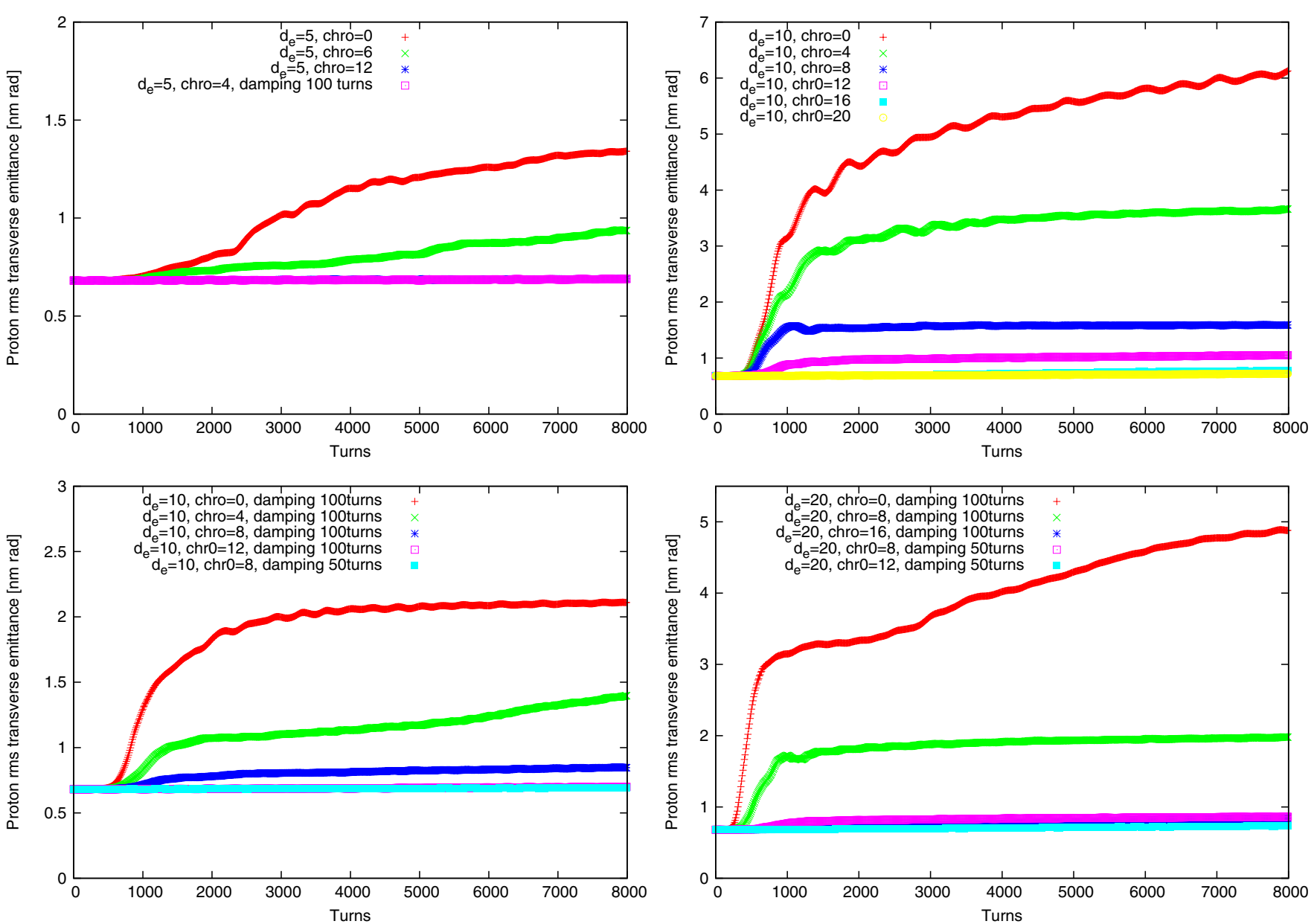

FIG. 5. The proton beam emittance growth due to the kink instability for different chromaticities at the disruption parameters 5 (in the top left figure) and 10 (in the top right figure). The top left figure also includes the case with transverse damper. The bottom left and bottom right figures correspond to the proton beam emittance growth with chromaticities together with transverse damper for $d_{e}=10$ and 20, respectively. The beam-beam parameters of the proton beam in all cases are 0.015 .

\section{FEEDBACK SCHEME OF KINK INSTABILITY}

To avoid undesirable nonlinear effects when suppressing the kink instability, we developed a dedicate feedback system as countermeasure of the transverse instability, including the transverse mode coupling instability (strong head-tail instability), and the head-tail instability due to longitudinal position dependence of the ion beam phase advance in that IR that was discussed in Sec. II.

The simple feedback system takes full advantage of flexibility of a linac-ring scheme, which has the following procedures. We steer the fresh electron bunch before collision based on the transverse offset of the last-used electron bunch that collides with the same ion bunch. Then the centroid of the new electron bunch will oscillate within the opposing ion bunch due to the focusing beam-beam force. We are expecting that oscillation of the centroid of the electron bunch gives the ion bunch proper kicks to correct the offset of the ion bunch before the visible adverse effect, such as emittance growth and luminosity loss, due to the kink instability. The schematic layout in Fig. 6 illustrates the general idea. For the ion beam, the "pickup" and "kicking" position of this feedback system are both at IP.

Mathematically, we introduce the offset by modifying the motion of the centroid of the electron bunch based on the information from the last one:

$$
\left(\begin{array}{c}
\bar{x}_{e} \\
\bar{x}_{e}^{\prime}
\end{array}\right)_{n+1, i}=M_{f}\left(\begin{array}{c}
\bar{x}_{e} \\
\bar{x}_{e}^{\prime}
\end{array}\right)_{n, f} .
$$

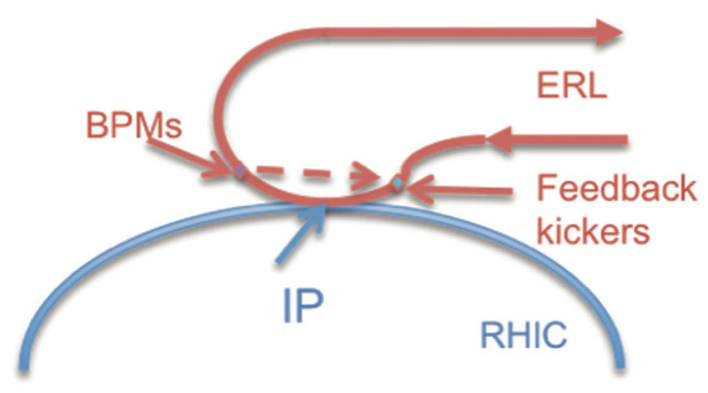

FIG. 6. The schematic layout of the feedback system for mitigating the kink instability in eRHIC. 
Here, the subscript $n$ denotes the electron-ion collision in the $n$th turn, the subscripts $i$ and $f$ respectively represent the bunch centroid before and after collision. Map $M_{f}$ defines the algorithm of the feedback system. Here, we limit $M_{f}$ to be a matrix for simplicity and easier realization. The propagation of electron bunch inside the ion bunch has an analytical solution if we assume the latter has a uniform longitudinal distribution with bunch length $2 L$, viz. $k^{2}(s)=1 /\left(L f_{e}\right)$, for a given initial condition with $\bar{x}_{e, 0}$ and $\bar{x}_{e, 0}^{\prime}$ at $s=L / 2$. The solution has two more terms from the initial condition than in Eq. (4) and becomes

$$
\begin{aligned}
\bar{x}_{e}= & k \int_{s}^{L / 2} \bar{x}_{i}\left(s^{\prime}, z=2 s^{\prime}\right) \sin k\left(s-s^{\prime}\right) d s^{\prime} \\
& +\bar{x}_{e, 0} \cos [k(L / 2-s)]-\bar{x}_{e, 0}^{\prime} \sin [k(L / 2-s)] / k
\end{aligned}
$$

in the collision region $[-L / 2, L / 2]$. In this simplified case, the two new terms have the wave number $k$, which is the same as the simplified wakefield form in Eq. (5). For other realistic longitudinal distributions of the ion beam, the trajectory of the electron bunch centroid will oscillate in a more complicate form and so must be calculated from the simulation. Undoubtedly, we require that the amplitude of the induced electron oscillation must be much smaller than the rms size of both colliding beams to prevent luminosity loss.

This oscillating term of the trajectory of the electron centroid will affect the ion bunch as described in Eq. (3). With proper feedback gain (the elements in feedback matrix $M_{f}$ ), the electron beam's oscillation will provide a longitudinal-position-dependent kick to the ion beam. The average kick over the longitudinal coordinates of the feedback system is similar to the effect of the bunch-by-bunch transverse damper. The longitudinal-position-dependent part of the kick is the key to suppress the instability, provided that its frequency component does not vanish at the frequency of the instability's dominant mode. We can use a simple model to illustrate the effect of the feedback system. We write the lowest head-tail mode of the ion bunch after the $n$th collision as $\bar{x}_{i}(s)=a_{n} \sin (\pi s / L)$, where $s=z / 2$ is the longitudinal position of the collision point and $a_{n}$ is the amplitude of this offset. If we measure the electron beam offset at $s=-L / 2$ as the input of the feedback system, the initial values for the next electron bunch induced by this offset can be calculate by using the head-tail mode ion offset $\bar{x}_{i}(s)$ in the integral of Eq. (17):

$$
\left(\begin{array}{c}
\bar{x}_{e, 0} \\
\bar{x}_{e, 0}^{\prime}
\end{array}\right)_{n+1}=\left(\begin{array}{c}
m_{11} \\
m_{21}
\end{array}\right) a_{n} D[1+\cos (\sqrt{D})] /\left(D-\pi^{2}\right)
$$

In the integral, we substitute $k L=\sqrt{D}$ to get simplified form. We note that the parameter $D$ actually is proportional to the disruption parameter. For a uniform longitudinal distribution in $[-L, L]$, the $\mathrm{rms}$ beam size $\sigma_{z}=L / \sqrt{3}$.

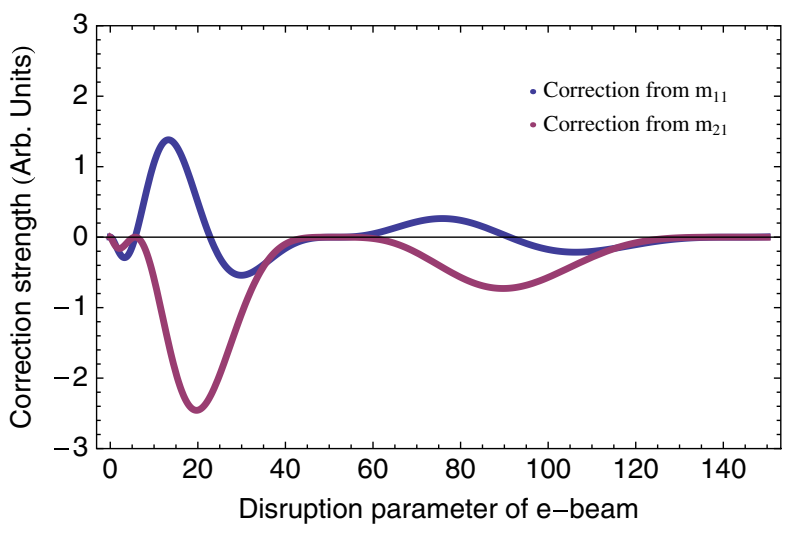

FIG. 7. The expected effectiveness of the proposed feedback system, calculated from the simplified model.

Therefore, $D=\sqrt{3} d_{e}$. The electron oscillation's Fourier component with the same frequency as the head-tail mode $\int x_{e, 0} \cos [k(L / 2-s)] \sin (\pi s / L)$ and $-\int \bar{x}_{e, 0}^{\prime} \times$ $\sin [k(L / 2-s)] \sin (\pi s / L)$ can be plotted in Fig. 7 as a function of the disruption parameter, assuming the headtail mode changes slowly $\left(\nu_{s} \ll 1\right)$. This component determines the effectiveness of corrections that the feedback system imposes on the lowest head-tail mode. From this simple model, we are anticipating the feedback system can communicate with the head-tail modes, and therefore can damp the instability with proper amplitude and sign of the feedback gain $\left(m_{11}\right.$ or $\left.m_{21}\right)$. However, at a certain range of disruption parameters (e.g. near $d_{e} \sim 6$ and $d_{e} \sim 55$ ), the efficiency of this feedback system for the lowest head-tail mode becomes zero from both oscillating terms. The effectiveness of the feedback system relies on two conditions. One is that the integral in Eq. (17) cannot vanish, which means the response of the electron beam to the ion offset of lowest mode can be detected at the beam position monitor (BPM) after collision. Second, the oscillating part of the same equation is not orthogonal to the ion beam offset, which indicates that the feedback induced oscillation can damp the existing offset of the ion beam. Therefore, in those ranges where the correction strength is zero, we expect that the instability cannot be damped using this method, which will be demonstrated in the below simulation results.

We will use the same simulation code to confirm our understanding of this idea. In the following examples, matrix $M_{f}$ has only one nonzero element $m_{11}$ to simplify the study and we keep the amplitude of the feedback system small, since a large amplitude will induce significant offset of the two colliding beams and cause luminosity loss. For two short colliding beams with round Gaussian transverse profile, the luminosity loss can be calculated as

$$
L=L_{0} \exp \left(-\frac{d^{2}}{2\left(\sigma_{1}^{2}+\sigma_{2}^{2}\right)}\right)
$$




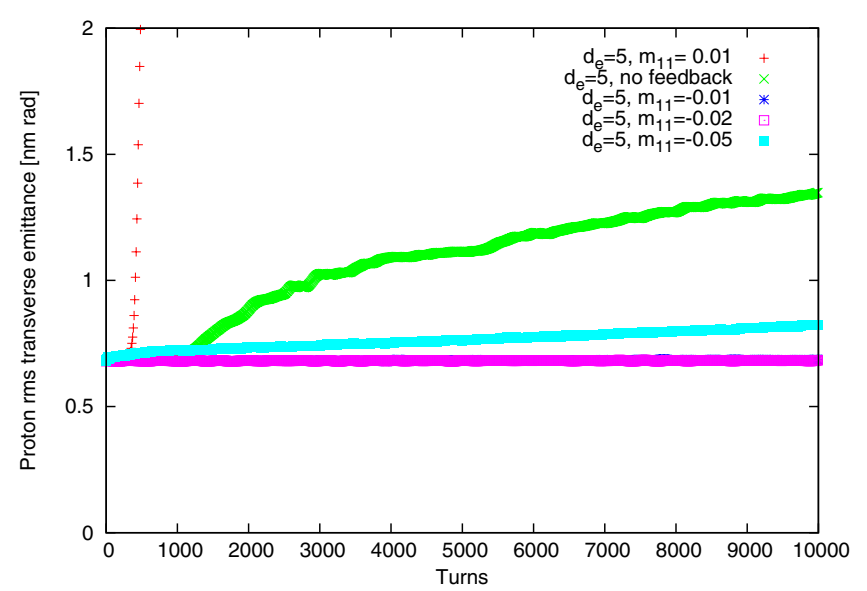

FIG. 8. The effect of the feedback system at disruption parameter 5 .

where $\sigma_{1,2}$ are the transverse sizes of two beams and $d$ is the displacement. We limit the gain of the feedback system so that the maximum oscillation amplitude of the electron beam does not exceeds $10 \%$ of the rms beam size, which corresponds to less than $0.5 \%$ luminosity loss. The chromaticity is hold at 2 units, viz., the nominal value in current RHIC operation. We assume the pickup BPM and the kicker are located at $L= \pm 3 \mathrm{~m}$, respectively. In all cases, we adjust the disruption parameters of the electron beam, while keeping the beam-beam parameter for the ion beam constant at 0.015 .

We will start our study with disruption parameter 5 to demonstrate the effect of this feedback system, although we already showed that the instability in this case could be suppressed by the bunch-by-bunch transverse damper with only a slight increment of chromaticity. In this $d_{e}=5$ case, we turned off the transverse damper in simulation to observe the pure effect of this feedback system. Figure 8 shows the emittance growth due to the kink instability is suppressed with proper amplitude of the feedback gain $m_{11}$ $(-0.01$ or -0.02$)$ without a noticeable decrease in luminosity. An incorrect sign of the gain may boost the instability, as shown by the red dots in Fig. 8. If we further increase the amplitude of the feedback gain, the effects of overcorrection on the ion beam appears, which also affects luminosity.

When the disruption parameter is close to 10 , we could not identify a proper feedback gain to stabilize the kink instability without reducing luminosity. More precisely, if the disruption parameter falls in the range of 9 to 13, the feedback system cannot damp the ion beam emittance growth due to the kink instability. The above analytical model predicts a smaller range because it uses different longitudinal distribution and a specific function for the lowest instability mode. Should the disruption parameter exceed this range $\left(d_{e}>13\right)$, the oscillation of the electron bunch will not only suppress the instability within the ion

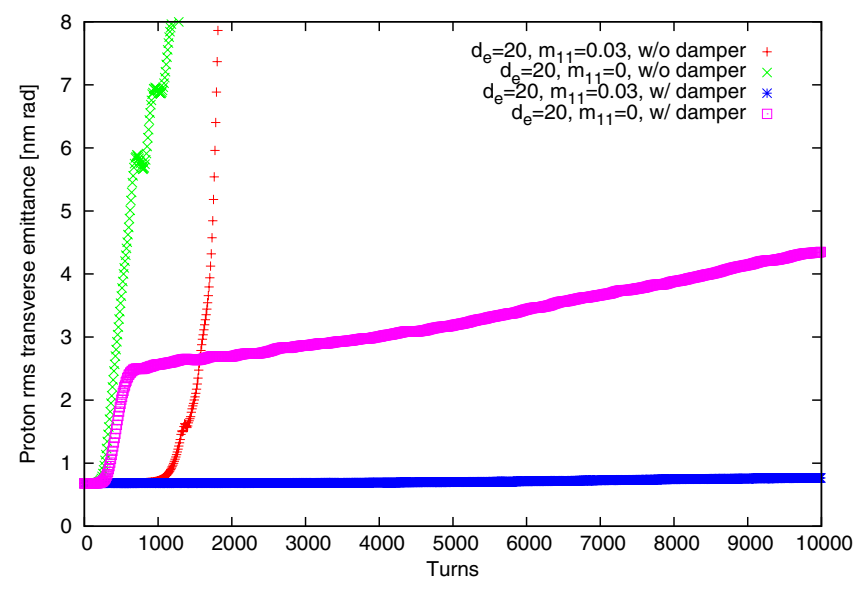

FIG. 9. The effect of the feedback system with or without the transverse damper at disruption parameter 20.

bunch, but also will destabilize the centroid of the entire ion bunch (mode zero or rigid mode). Therefore, this feedback system must be applied together with the bunch-bybunch transverse damper. In this $d_{e}$ range, we turned on the transverse damper with the assumption of damping time of 100 turns in the simulation. The result for $d_{e}=20$, as an example, is shown in Fig. 9. The red dots show the case with the feedback gain of $m_{11}=0.03$ without transverse damper. The centroid of the ion bunch becomes unstable and causes fast emittance growth due to the offset of two beams. By applying the bunch-by-bunch feedback in the simulation, the ion centroid is stable and the emittance growth is prevented (blue curve).

The feedback system shows promising results in the above range of disruption parameter. However, a limitation is inevitable for such a simple system. If we further increase the disruption parameter $\left(d_{e} \geq 25\right)$, the efficiency of the feedback system starts decreasing. Therefore to suppress the emittance growth, it requires larger gains, which lead to large amplitudes of electron beam oscillation. The large amplitude is harmful in two ways. First, large amplitude will push the correction force towards the nonlinear regime and induce additional emittance growth; second, it will lead to significant luminosity loss of the electron-ion collision. For the case $d_{e}=25$, minimum gain to suppress the instability is $m_{11}=0.05$, with $10 \%$ luminosity loss, while, for $d_{e}=30$, the luminosity loss becomes $40 \%$ and the emittance growth cannot be fully suppressed for the optimum gain $m_{11}=0.05$. In simulation, we also tested the effectiveness of the $m_{21}$, which gives similar limitation as $m_{11}$.

Despite these drawbacks, this simple scheme offers an effective and promising way to resolve the kink instability in a large range of disruption parameter in which eRHIC parameters reside. In those working ranges, the system can suppress the emittance growth of the opposing ion beam at very small chromaticity ( +2 units) 
without observable luminosity loss. For those unresolved ranges of disruption parameter, a traditional broadband pickup-kicker feedback system with proper bandwidth is needed in the ion ring. We will present the requirement of such a system in another paper; our preliminary studies are given in [11].

\section{SUMMARY}

In this paper, we reviewed the origin of the kink instability, the unique two-beam instability that linacring-type EICs are prone to developing. As an example, parameters of the EIC proposed at BNL, eRHIC, exceeds the threshold of strong head-tail instability. The chromaticity, together with a transverse damper, can stabilize the emittance growth due to the instability only when it is set to unacceptably large values. Instead, feedback systems are suitable as countermeasures. A simple feedback system on the electron beam together with a transverse bunch-by-bunch feedback system can be implemented to suppress the instability up to the e-beam disruption parameter 25 , provided that the beam-beam parameter for the ion beam is kept as 0.015. However, this method becomes less effective for larger disruption parameters and over a small range of $d_{e}$ (9-13) when the efficiency of the feedback system vanishes, according to our simulation with eRHIC parameters. A traditional broadband pickup-kicker feedback system that can damp the instability coherently is needed to damp those disruption parameter ranges.

\section{ACKNOWLEDGMENTS}

The author would like to thank Michael Blaskiewicz and Yun Luo for their constructive help and suggestions. This work is supported by the U.S. Department of Energy under Contract No. DE-AC02-98CH10886.

[1] V. Ptitsyn et al., Technical Report, C-AD, BNL, 2007.

[2] Y. Hao and V. Ptitsyn, Phys. Rev. ST Accel. Beams 13, 071003 (2010).

[3] R. Li and J. J. Bisognano, Phys. Rev. E 48, 3965 (1993).

[4] R. Li, B. Yunn, V. Lebedev, and J. Bisognano, in Proceedings of the Particle Accelerator Conference, Chicago, IL, 2001 (IEEE, New York, 2001).

[5] Y. Hao, Ph.D. thesis, Indiana University, 2008.

[6] A. W. Chao, Physics of Collective Beam Instabilities in High Energy Accelerators (Wiley-Interscience, New York, 1993).

[7] B. Yunn, Jlab Technical Report No. JLAB-TN-01-017.

[8] V. Danilov and E.A. Perevedentsev, Nucl. Instrum. Methods Phys. Res., Sect. A 391, 77 (1997).

[9] V. Ptitsyn, Y. Hao, and V. Litvinenko, in Proceedings of HB2010, Morschach, Switzerland, 2010 (PSI, Viligen, Switzerland, 2011).

[10] E. A. Perevedentsev and A. A. Valishev, Phys. Rev. ST Accel. Beams 4, 024403 (2001).

[11] Y. Hao, M. Blaskiewicz, V. Litvinenko, and V. Ptitsyn, in Proceedings of the 3rd International Particle Accelerator Conference, New Orleans, Louisiana, 2012 (IEEE, Piscataway, NJ, 2012). 